Henian Cao - Andrea Mok • Brooke Miskie

Robert A. Hegele

\title{
Single-nucleotide polymorphisms of the proprotein convertase subtilisin/ kexin type 5 (PCSK5) gene
}

Received: July 23, 2001 / Accepted: August 28, 2001

\begin{abstract}
The proprotein convertase, subtilisin/kexin type 5 , or PCSK5, mediates post-translational endoproteolytic processing for several integrin $\alpha$ subunits. We identified two silent single-nucleotide polymorphisms (SNPs) in PCSK5, which were found to vary in frequency across ethnic groups. The identification of these amplification primers and SNPs provides tools to investigate $P C S K 5$ for association with inflammatory or vascular phenotypes.
\end{abstract}

Key words Inflammation · Cardiovascular disease Genomic DNA · Sequencing $\cdot$ Complex traits

\section{Introduction}

Proprotein convertases are calcium-dependent serine proteases related to bacterial subtilisins and to yeast kexin (Seidah and Chretien 1999). These enzymes process precursor proteins to their active forms by selective cleavage of the polypeptide at sites following paired basic amino acids. In mammals, this family comprises PCSK1, PCSK2, PCSK4, PCSK5, furin, and PACE4 (Seidah and Chretien 1999). Substrates for these enzymes range from precursors for growth factors to cell surface receptors and viral surface glycoproteins. For instance, several integrin $\alpha$ subunits undergo post translational endoproteolytic processing at pairs of basic amino acids that is mediated by the proprotein convertase, subtilisin/kexin type 5, or PCSK5 (Lissitzky et al. 2000). PCSK5 (OMIM \#600488) encodes this proprotein convertase, which has been mapped to 9q21.3 (van de Loo et al. 1996). We used our established approach (Cao and Hegele 2000) to develop amplification primers for PCSK5 coding sequences from genomic DNA and report the

H. Cao · A. Mok · B. Miskie $\cdot$ R.A. Hegele $(\bowtie)$

Blackburn Cardiovascular Genetics Laboratory, John P. Robarts Research Institute, 406-100 Perth Drive, London, ON N6A 5K8, Canada

Tel. +1-519-663-3461; Fax +1-519-663-3789

e-mail: robert.hegele@rri.on.ca identification of two single-nucleotide polymorphisms (SNPs).

\section{Methods}

Study subjects

We screened genomic DNA from samples taken from 16 normal unrelated subjects. To estimate population frequencies of the PCSK5 SNPs, we screened 226 alleles from clinically normal subjects from various ethnic groups $(66$ Caucasian, 60 Chinese, and 50 each of African and Amerindian). The study was approved by the Ethics Review Panel, University of Western Ontario.

Screening the PCSK5 gene for DNA variants

To amplify coding regions and intron-exon boundaries from genomic DNA, we developed a primer set using GenBank sequences (see Table 1). Primer sequences were derived using GenBank accession numbers XM_011818 and 13641334. Primers were designed to anneal at a single temperature, which allowed for use of a single amplification apparatus. Amplification conditions were $94^{\circ} \mathrm{C}$ for $5 \mathrm{~min}$, followed by 30 cycles of $30 \mathrm{~s}$ each at $94^{\circ} \mathrm{C}, 58^{\circ} \mathrm{C}$, and $72^{\circ} \mathrm{C}$, and ending with a single 10 -min extension step at $72^{\circ} \mathrm{C}$. Amplified fragments were purified and directly sequenced (ABI 377 Prism, ABI, Mississauga, ON, Canada).

Genotyping of PCSK5 gene SNPs

The PCSK5 1191G > A SNP was genotyped using amplification with primers for exon 3 , as shown in Table 1 . The resulting fragment was $350 \mathrm{bp}$ in length. Digestion of the $1191 \mathrm{G}$ allele with $D d e$ I produced four fragments with length $221,75,31$, and $23 \mathrm{bp}$, whereas digestion of the $1191 \mathrm{~A}$ allele produced five fragments, with length 119,102 , 75,31 , and $23 \mathrm{bp}$. These fragments were resolved by electrophoresis in $2 \%$ agarose gels. 
Table 1. Amplification primers for PCSK5

\begin{tabular}{|c|c|c|}
\hline Exon & Primer sequence & Product size (bp) \\
\hline \multirow[t]{2}{*}{ Exon 1} & F 5'- ATT CTT ACC TTG TCT TTC CCC A $-3^{\prime}$ & 260 \\
\hline & R 5'- AAA GAT CAA GCT CTT TGT TTG TCA -3' & \\
\hline \multirow[t]{2}{*}{ Exon 2} & F 5'- TGA GTG ACG TTA TAA TTT GAC CTA TG -3' & 227 \\
\hline & R 5'- CTC TAT TTT GGG GCA TTG TCA T -3' & \\
\hline \multirow[t]{2}{*}{ Exon 3} & F 5'- TAT TTA TTC CAT GCC TCC AGG T -3' & 350 \\
\hline & R 5'- AGC AGC TGT TCC CTT CTA CCT A -3' & \\
\hline \multirow[t]{2}{*}{ Exon 4} & F 5'- CAT TGG ATT TGA AAT ATC GCA C - $3^{\prime}$ & 251 \\
\hline & R 5'- CTG GCC AAA ATA TAC TCC ATC A -3' & \\
\hline \multirow[t]{2}{*}{ Exon 5} & F 5'- TTG GTC ATG CTG TGA TTT CAT T -3' & 274 \\
\hline & R 5'- TTT CAT TCC ATT CCA TTC CAT T -3' & \\
\hline \multirow[t]{2}{*}{ Exon 6} & F 5' - ATT CAA GGT AAA GCT GAC CTG C -3' & 255 \\
\hline & R 5'- GCA CAC ACC CCA TGG TAT TAG -3' & \\
\hline \multirow[t]{2}{*}{ Exon 7} & F 5'- CTC AGC ACC TCC AAG AGA ATG - $3^{\prime}$ & 332 \\
\hline & R 5'- GGT CAC TCA AAT GAA AAC ACC A -3' & \\
\hline \multirow[t]{2}{*}{ Exon 8} & F 5'- TGC TTT TAT GGA ATC CAA TTG AC -3' & 200 \\
\hline & R 5'- ATT TAC ATT GAG AAG CTC TTT GGG -3' & \\
\hline \multirow[t]{2}{*}{ Exon 9} & F 5'- AGT CAT TAC GTT TTG GCC TCA T -3' & 223 \\
\hline & R 5'- CCA AAC CAG AAA GAA AAG CAA A -3' & \\
\hline \multirow[t]{2}{*}{ Exon 10} & F 5'- ATT CTT TGA CGC CAT TTT CTC A -3' & 250 \\
\hline & R 5'- AGG GGG CAG ATC CAC TTA CT -3' & \\
\hline \multirow[t]{2}{*}{ Exon 11} & F 5'- AGT AAA CAC ACC TTC CCC TGT G -3' & 256 \\
\hline & R 5'- AAG TGG AAA AAT ATT AGT CCT GAA AG -3' & \\
\hline \multirow[t]{2}{*}{ Exon 12} & F 5'- TTC CCC AAA TCT GCC TCT CT -3' & 250 \\
\hline & R 5'- ACA GGA ACA AAG AAA TCT AAG ATG G -3' & \\
\hline
\end{tabular}

The PCSK5 2007C > T SNP was genotyped by amplification with primers for exon 9, as shown in Table 1. The resulting fragment was $223 \mathrm{bp}$ in length. Digestion of the 2007C allele with Alw21I produced two fragments with length 141 and $82 \mathrm{bp}$, whereas digestion of the 2007T allele produced one fragment, with length $223 \mathrm{bp}$. These fragments were resolved after electrophoresis in $2 \%$ agarose gels.

Statistical analysis

SAS version 6.12 (SAS Institute, Cary, NC, USA) was used for statistical analyses. Allele frequencies were determined from electropheretogram tracings of genomic DNA sequence, except for two SNPs that were assayed using restriction digestion. Chi-square analysis tested the deviation of genotype frequencies from Hardy-Weinberg predictions. The nominal level of significance for statistical analyses was $P<0.05$.

\section{Results}

SNP identification

Genomic DNA sequencing found two silent coding SNPs, designated PCSK5 1191G $>\mathrm{A}$ in exon 3, and 2007C $>\mathrm{T}$ in exon 9. No other variants were found.

\section{SNP frequencies}

The observed genotype frequencies of all SNPs did not deviate from Hardy-Weinberg expectations, and allele fre-
Table 2. $P C S K 5$ gene single-nucleotide polymorphism allele frequencies

\begin{tabular}{llll}
\hline Sample & Number & $\begin{array}{l}\text { Exon 3 1191A } \\
\text { frequency }\end{array}$ & $\begin{array}{l}\text { Exon 9 2007T } \\
\text { frequency }\end{array}$ \\
\hline African & 50 & 0.16 & 0.04 \\
Caucasian & 66 & 0.27 & 0.17 \\
Chinese & 60 & 0.38 & 0.00 \\
Amerindian & 50 & 0.41 & 0.00 \\
\hline
\end{tabular}

quencies are shown in Table 2. The PCSK5 exon 9 2007T allele was absent from Chinese and Amerindian study samples.

\section{Discussion}

We report (1) the definition of primer sets to amplify the coding sequences of the PCSK5 gene; and (2) the use of these amplification primers for genomic DNA sequencing, which has resulted in (3) the identification of two novel SNPs. The reagents described in this report could be helpful to screen the PCSK5 gene when it becomes a candidate for phenotypes that involve inflammation or the cardiovascular system, through positional cloning, analogy with other phenotypes, or expression data showing changes in response to particular experimental interventions. The SNPs could also be helpful in linkage mapping and for association studies.

Acknowledgements Pearl Campbell provided outstanding technical assistance. Dr. Hegele holds a Canada Research Chair in Human Genetics and a Career Investigator award from the Heart and Stroke Foundation of Ontario. This work was supported by grants from the 
Canadian Institutes for Health Research (MT13430), the Heart and Stroke Foundation of Ontario (T4772), the Canadian Genetic Diseases Network, and the Blackburn Group.

\section{References}

Cao H, Hegele RA (2000) Nuclear lamin A/C R482Q mutation in Canadian kindreds with Dunnigan-type familial partial lipodystrophy. Hum Mol Genet 9:109-112
Lissitzky JC, Luis J, Munzer JS, Benjannet S, Parat F, Chretien M, Marvaldi J, Seidah NG (2000) Endoproteolytic processing of integrin pro- $\alpha$ subunits involves the redundant function of furin and proprotein convertase (PC) $5 \mathrm{~A}$, but not paired basic amino acid converting enzyme (PACE) 4, PC5B or PC7. Biochem J 346:133-138 Seidah NG, Chretien M (1999) Proprotein and prohormone convertases: a family of subtilases generating diverse bioactive polypeptides. Brain Res 848:45-62

van de Loo J-WHP, Creemers JWM, Kas K, Roebroek AJM, Van de Ven WJM (1996) Assignment of the human proprotein convertase gene PCSK5 to chromosome 9q21.3. Cytogenet Cell Genet 75:227229 